\title{
The Effects of Excluding Coalitions
}

\author{
Tobias Hiller ${ }^{1,2}$ \\ 1 Institute for Theoretical Economics, University of Leipzig, D-04109 Leipzig, Germany; \\ hiller@wifa.uni-leipzig.de \\ 2 HR Department, TU Dresden, D-01062 Dresden, Germany
}

Received: 2 November 2017; Accepted: 18 December 2017; Published: 1 January 2018

\begin{abstract}
One problem in cooperative game theory is to model situations when two players refuse to cooperate (or the problem of quarreling members in coalitions). One example of such exclusions is the coalition statements of parliamentary parties. Other situations in which incompatible players affect the outcome are teams in firms and markets, for example. To model these exclusions in cooperative game theory, the excluded coalitions value ( $\varphi^{E}$ value) was introduced. This value is based on the Shapley value and takes into account that players exclude coalitions with other players. In this article, we deduce some properties of this new value. After some general results, we analyze the apex game that could be interpreted as a team situation and the glove game that models markets where sellers and buyers deal. For team situations, we show that all employees have a common interest for cooperation. On asymmetric markets, excluding coalitions affect the market players of the scarce side to a higher extent.
\end{abstract}

Keywords: excluded coalitions; quarreling; $\varphi^{E}$ value; Shapley value; cooperative game theory

\section{Introduction}

One problem in cooperative game theory is to model situations when two players refuse to cooperate (or the problem of quarreling members in coalitions). One example of such exclusions is the coalition statements of parliamentary parties. Other situations in which incompatible players affect the outcome are teams in firms and markets, for example. To model this, the $\varphi^{E}$ value (excluded coalitions value) for cooperative games was introduced and axiomatized [1]. This value is based on the Shapley value [2] and takes into account that players exclude coalitions with other players. The $\varphi^{E}$ value enhances the approaches developed by [3] to model that players prefer some other players for cooperation. ${ }^{1}$ Whereas the approach by $[3,5]$ modifies the coalitional function of the game, the model by restricts the set of admissible permutations of players. For modeling the preferences of players, both models are insufficient. Assume a parliament with three parties. One party is located on the left, one party is on the right and one party is in the middle. The left and the right parties exclude coalitions with each other. The party from the middle admits cooperation with both parties. In the models by $[3,5]$ the left and the right parties are connected via the middle party. Hence, all parties cooperate. Using the $\varphi^{E}$ value, the excluded coalition between the left and the right players is considered in a way that precludes a coalition of all three players.

1 Another enhancement of the approach by [3] was introduced by [4]. In this model, incompatible players are linked by an arc of the graph. As well as in the models by [3,5] , every coalition (especially the grand coalition) obtains a worth-the maximum that could achieved by the compatible players of the coalition. In the model by [4], loops or parallel arcs are not intended. This limitation does not exist when calculating the $\varphi^{E}$ value. In addition, a further analysis of stable coalition structures based on the excluded coalition partners $[6,7]$ may be complicated if the worth of the grand coalition, for example, is a superadditive hull. 
The articles by [8,9] analyzed situations where each player could decide to exclude coalitions to all other players. Using exclusions of coalitions strategically was analyzed by $[10,11]$. In these articles, a non-cooperative game models the strategic decision of players whereas the values of cooperative game theory determine the payoffs at the last stage.

Since the $\varphi^{E}$ value is a new value, there are many fields of research with fruitful questions. One major question is how excluding of coalitions influences the players' payoffs. For weighted majority games, this question was addressed in [1]. In this article, we deduce the influence of excluding coalitions on the players' $\varphi^{E}$ payoffs. We start with some general games (monotone games) and, in the final stage, we analyze the apex game that could be interpreted as a team situation and glove games that models markets where sellers and buyers deal. For team situations, we show that all employees have a common interest for cooperation. On asymmetric markets, excluding coalitions affect the market players of the scarce side to a higher extent.

The remainder of this article is structured as follows. Basic definitions of cooperative game theory are presented in Section 2. In Section 3, we present our results. Section 4 concludes.

\section{Basic Notation}

A game is a pair $(N, v)$ and $N=\{1,2, \ldots, n\}$ is the player set. The coalitional function $v$ assigns every subset $K$ of $N$ a certain worth $v(K)$, reflecting the economic abilities of $K$ (i.e., $v: 2^{N} \rightarrow \mathbb{R}$ ) such that $v(\varnothing)=0$. A game is called monotone if $v(T)<v(S)$, for all $T \subset S, T, S \subseteq N$. A game is convex if $v(T \cup\{i\})-v(T)<v(S \cup\{i\})-v(S)$, for all $T \subset S, T, S \subseteq N$ and $i \notin S$. A game $(N, v)$ is designated symmetric or anonymous, if a function $f: N \rightarrow \mathbb{R}$ exists such that $v(K)=f(|K|)$ for all non-empty sets $K \subseteq N$. Hence, the number of players determines the worth of a coalition. The cardinality of $N$ is called $n$ or $|N|$.

One important value for games $(N, v)$ is the Shapley value. To calculate the player's payoffs, rank orders $\rho$ on $N$ are used. They are written as $\left(\rho_{1}, \ldots, \rho_{n}\right)$ where $\rho_{1}$ is the first player in the order, $\rho_{2}$ the second player, etc. The player at position $t$ is noted by $\rho(t)$. The set of these orders is denoted by $R O(N) ; n$ ! rank orders exist. The set of players before $i$ in rank order $\rho$ together with player $i$ is called $P_{i}(\rho)$. The Shapley payoff of a player $i$ is the average of the marginal contributions of $i$ taken over all rank orders of the players:

$$
\varphi_{i}(N, v)=\frac{1}{n !} \sum_{\rho \in R O(N)} v\left(P_{i}(\rho)\right)-v\left(P_{i}(\rho) \backslash\{i\}\right) .
$$

The $\varphi^{E}$ value is based on the Shapley value. For the calculation of the $\varphi^{E}$ value, we take into account the statements of the players on excluded coalitions. The set of $i^{\prime}$ s excluded coalition partners is denoted by $E_{i}$. A player excludes only coalitions to single players; $|K|=1, K \in E_{i}$. The set of coalitions that are not allowed based on $E_{i}$ is called $X_{i}, X_{i}:=\left\{K \subseteq N \mid K \backslash\{i\} \in E_{i}\right\}$ with $|K|=2$. If $i$ does not cooperate with $j$, we have $X_{i} \cap X_{j}=\{i, j\}$, i.e., if a player $i$ does not cooperate with $j$, also $j$ cannot cooperate with $i$. All inadmissible coalitions are denoted by $\Gamma:=\left\{K \subseteq N \mid \exists S \in X_{j}, j \in N\right.$, with $\left.S \subseteq K\right\}$. Thus, the admissible coalitions in the game $(N, v)$ are $\Omega:=\{K \subseteq N \mid K \notin \Gamma\}$. The set of admissible coalitions containing player $i$ is denoted by $\Omega(i)$. The tuple $(N, v, \Gamma)$ is a game with excluded cooperation partners.

Example 1. Let $N=\{1,2,3\}, E_{1}=\{\{2\},\{3\}\}, E_{2}=\{\{1\}\}$ and $E_{3}=\{\{1\}\}$. From this, we obtain the following sets $X_{i}: X_{1}=\{\{1,2\},\{1,3\}\}, X_{2}=\{\{1,2\}\}, X_{3}=\{\{1,3\}\}$. From these sets, we deduce the set of inadmissible coalitions $\Gamma=\{\{1,2\},\{1,3\},\{1,2,3\}\}$ and the set of admissible coalitions $\Omega=\{\varnothing,\{1\},\{2\},\{3\},\{2,3\}\}$.

The $\varphi^{E}$ value is one value for games with excluded cooperation partners. The primary idea of the $\varphi^{E}$ value is that only marginal contributions to admissible coalitions influence the players' payoffs. All supersets of excluded coalitions are inadmissible. 
Hence, the $\varphi^{E}$ payoff for player $i$ in $(N, v, \Gamma)$ is calculated by [1]:

$$
\varphi_{i}^{E}(N, v, \Gamma)=\frac{1}{n !} \sum_{\rho \in R O(N), P_{i}(\rho) \in \Omega} v\left(P_{i}(\rho)\right)-v\left(P_{i}(\rho) \backslash\{i\}\right) .
$$

In the case of $\Gamma=\varnothing$, we have $\varphi_{i}^{E}(N, v, \Gamma)=\varphi_{i}(N, v)$.

\section{Results}

In this section, first we present some results on how excluding of coalitions affects the players' payoffs. We start our analysis with general games like monotone games or symmetric games. After this, we analyze apex games and glove games. Results on weighted majority games were drawn in [1].

For monotone games, we deduce from Equation (2):

Corollary 1. Let $(N, v, \Gamma)$ be a monotone game with excluded cooperation partners. For $i, j \in N, i \neq j$ such that neither $i \in E_{j}$ nor $j \in E_{i},\{i, j\} \notin \Gamma$, we have

$$
\varphi_{i}^{E}(N, v, \Gamma) \geq \varphi_{i}^{E}\left(N, v, \Gamma^{\prime}\right)
$$

where $\{i, j\} \in \Gamma^{\prime}$.

Hence, excluding coalitions reduces $i^{\prime} \mathrm{s} \varphi^{E}$ payoff in monotone games.

In addition, we deduce from Equation (2) the next Corollary:

Corollary 2. Let $(N, v, \Gamma)$ be a convex game with excluded cooperation partners. For $i, j, l \in N, i \neq j \neq l$ such that neither $i \in E_{j}$ nor $j \in E_{i},\{i, j\} \notin \Gamma$, and neither $i \in E_{l}$ nor $l \in E_{i},\{i, l\} \notin \Gamma$, we have

$$
\varphi_{i}^{E}(N, v, \Gamma)-\varphi_{i}^{E}\left(N, v, \Gamma^{\prime}\right)>\varphi_{i}^{E}\left(N, v, \Gamma^{\prime}\right)-\varphi_{i}^{E}\left(N, v, \Gamma^{\prime \prime}\right)
$$

where $\{i, l\} \in \Gamma^{\prime},\{i, l\} \in \Gamma^{\prime \prime}$ and $\{i, j\} \in \Gamma^{\prime \prime}$.

Hence, excluding the "first" player affects the $\varphi^{E}$ payoff of player $i$ more than excluding one more player.

With an example we show that superadditivity ${ }^{2}$ is not sufficient. Assume a game with excluded cooperation partners with $N=\{1,2,3\}, v(\{1\})=0, v(\{2\})=2, v(\{3\})=1, v(\{1,2\})=2$, $v(\{1,3\})=4, v(\{2,3\})=5, v(N)=6$ and $\Gamma=\varnothing$. The game is superadditive but not convex. Table 1 shows the marginal contributions and the resulting $\varphi^{E}$ payoff for player 1 . In a first step, cooperation with player 2 is excluded. In the next step (last column), cooperation with 3 is excluded additionally.

Table 1. superadditive game with excluded coalitions.

\begin{tabular}{cccc}
\hline $\boldsymbol{R O}(\boldsymbol{N})$ & $\Gamma=\varnothing$ & $\Gamma=\{\{\mathbf{1}, \mathbf{2}\}, N\}$ & $\Gamma=\{\{\mathbf{1}, \mathbf{2}\},\{\mathbf{1}, \mathbf{3}\}, N\}$ \\
\hline $1,2,3$ & 0 & 0 & 0 \\
$1,3,2$ & 0 & 0 & 0 \\
$2,1,3$ & 0 & - & - \\
$2,3,1$ & 1 & - & - \\
$3,1,2$ & 3 & 3 & - \\
$3,2,1$ & 1 & - & - \\
\hline$\varphi_{1}^{E}$ & $\frac{5}{6}$ & $\frac{3}{6}$ & $\frac{0}{6}$ \\
\hline
\end{tabular}

2 A game is superadditive if $v(S)+v(T) \leq v(S \cup T), T \cap S=\varnothing, T, S \subseteq N$ 
In the next step, we analyze symmetric games. From Theorem 16 in [1], we deduce the following Corollary:

Corollary 3. Let $(N, v, \Gamma)$ be a symmetric game with excluded cooperation partners. For $i, j \in N, i \neq j$ such that neither $i \in E_{j}$ nor $j \in E_{i},\{i, j\} \notin \Gamma$, we have

$$
\varphi_{i}^{E}(N, v, \Gamma)-\varphi_{i}^{E}\left(N, v, \Gamma^{\prime}\right)=\varphi_{j}^{E}(N, v, \Gamma)-\varphi_{j}^{E}\left(N, v, \Gamma^{\prime}\right) .
$$

where $\{i, j\} \in \Gamma^{\prime}$.

Both players' $\varphi^{E}$ payoffs are affected in the same way. Corollary 3 is similar to properties of other values like the balanced contributions axiom of the Shapley value [12], the property of fair gain from bilateral links [3] or the splitting axiom of the $\chi$ value [7]. Corollary 4 is deduced from Theorem 17 in [1]. It presents the impact of the number of excluded coalitions in a symmetric game.

Corollary 4. Let $(N, v, \Gamma)$ be a monotone symmetric game with excluded cooperation partners with $n>2$. For $i, j \in N, i \neq j$, such that $\left|E_{i}\right|>\left|E_{j}\right|$ we have

$$
\varphi_{j}^{E}(N, v, \Gamma)>\varphi_{i}^{E}(N, v, \Gamma) .
$$

Hence, players with a higher number of excluded coalitions have lower $\varphi^{E}$ payoffs than do players with less number of excluded coalitions.

The apex game was introduced by [13]. An overview about the existing literature on apex games is provided by [14]. The apex game is defined for $n \geq 2$. There is one apex player $i_{0}$. The other players are minor ones. All coalitions which contain $i_{0}$ and at least one minor player as well the coalition which contains all minor players get the worth of 1 while all other coalitions get zero. This game could be interpreted as a team situation in firms where $i_{0}$ is the manager. He needs at least one team member to create a worth.

Theorem 1. Let $(N, v, \Gamma)$ be an apex game with excluded cooperation partners and $E_{i}=\varnothing$ for all $i \in N$. For all $l, m \in N \backslash\left\{i_{0}\right\}, l \neq m$, we have

$$
\varphi_{l}^{E}(N, v, \Gamma)-\varphi_{l}^{E}\left(N, v, \Gamma^{\prime}\right)=\varphi_{m}^{E}(N, v, \Gamma)-\varphi_{m}^{E}\left(N, v, \Gamma^{\prime}\right)
$$

where $\{i, j\} \in \Gamma^{\prime}, i, j \in N \backslash\left\{i_{0}\right\}$.

Proof. Without excluded coalitions, the players' $\varphi^{E}$ payoffs are:

$$
\begin{aligned}
\varphi_{i_{0}}^{E}(N, v, \Gamma) & =\varphi_{i_{0}}(N, v)=\frac{n-2}{n} \\
\varphi_{l}^{E}(N, v, \Gamma) & =\varphi_{l}(N, v)=\frac{1-\frac{n-2}{n}}{n-1}=\frac{2}{n(n-1)}
\end{aligned}
$$

with $l \in N \backslash\left\{i_{0}\right\}$. The player $i_{0}$ does not obtain the marginal contribution 1 in the rank orders at positions 1 and $n$. In $n-2$ from $n$ possible positions, he obtains the marginal contribution 1 . The remaining marginal contributions $1-\frac{n-2}{n}$ are divided equally to the minor players. Excluding a coalition between $i, j \in N \backslash\left\{i_{0}\right\}$ prevents the possibility for all minor players, to obtain the marginal contribution 1 at position $n-1$. Since all minor players are symmetric, their $\varphi^{E}$ payoffs are affected to the same extent. The possibility to obtain the marginal contribution 1 at the second position, if player $i_{0}$ is at first position, is unchanged for the minor players. 
Excluding coalitions between $j$ and $i$ affects the $\varphi^{E}$ payoffs of all players $l \in N \backslash\left\{i_{0}\right\}$ in the same way. Hence, all minor players have a common interest that all of them are willing to cooperate. The next Corollary follows directly:

Corollary 5. Let $(N, v, \Gamma)$ be an apex game with excluded cooperation partners with $i, j \in N \backslash\left\{i_{0}\right\}, i \neq j$, such that $i \in E_{j}$ (and/or $j \in E_{i}$ ). For all $l \in N \backslash\left\{i_{o}\right\}$ we have

$$
\varphi_{l}^{E}(N, v, \Gamma)=\varphi_{l}^{E}\left(N, v, \Gamma^{\prime}\right)
$$

where $\{i, m\} \in \Gamma^{\prime}, m \in N \backslash\left\{i_{0}, i, j\right\}$ and $i \in N \backslash\left\{i_{o}\right\}$.

After excluding a coalition between $i, j \in N \backslash\left\{i_{o}\right\}$, the minor players obtain only a marginal contribution 1 at the second position, if player $i_{0}$ is at first position. With excluding further coalitions between minor players this possibility is not affected. Hence, if one coalition between minor players is excluded, further exclusions of coalitions between the minor players do not change their $\varphi^{E}$ payoffs.

The last game analyzed is the so-called glove game [15]. In this game, each player owns either one left glove or one right glove. A single glove has no worth; a pair of gloves has a worth of one. Hence, this game models markets where sellers and buyers deal. The coalitional function for this game is given by

$$
v(K)=\min \{|K \cap L|,|K \cap R|\} \text { with } N=R \cup L, R \cap L=\varnothing,
$$

where $L(R)$ denotes the set of left (right) glove owners. The worth of a coalition equals the number of matching pairs it contains.

Corollary 6. Let $(N, v, \Gamma)$ be a glove game with excluded cooperation partners with $|R|=|L|$ and $E_{i}=\varnothing$ for all $i \in N$. For $i, j \in N, i \neq j$ with $i \in L, j \in R$ we have

$$
\varphi_{i}^{E}(N, v, \Gamma)-\varphi_{i}^{E}\left(N, v, \Gamma^{\prime}\right)=\varphi_{j}^{E}(N, v, \Gamma)-\varphi_{j}^{E}\left(N, v, \Gamma^{\prime}\right)
$$

where $\{i, j\} \in \Gamma^{\prime}$.

Without excluded coalitions, the players' $\varphi^{E}$ payoffs are:

$$
\varphi_{j}^{E}(N, v, \Gamma)=\varphi_{i}^{E}(N, v, \Gamma)=\varphi_{i}(N, v)=\frac{1}{2} .
$$

Both types of players are symmetric. Excluding coalitions between $j$ and $i, i \in L, j \in R$, affects both players to the same extent; they stay symmetric. Hence, on symmetric markets with an equal number of players on each market side, excluding a coalition with a player from the opposite side of the market reduces the players' $\varphi^{E}$ payoffs in an equal way.

Now, we analyze asymmetric glove games with $|R|<|L|$ :

Theorem 2. Let $(N, v, \Gamma)$ be a glove game with excluded cooperation partners with $|R|<|L|$ and $E_{i}=\varnothing$ for all $i \in N$. For $i, j \in N, i \neq j$ with $i \in L, j \in R$ we have

$$
\varphi_{j}^{E}(N, v, \Gamma)-\varphi_{j}^{E}\left(N, v, \Gamma^{\prime}\right)>\varphi_{i}^{E}(N, v, \Gamma)-\varphi_{i}^{E}\left(N, v, \Gamma^{\prime}\right)
$$

where $\{i, j\} \in \Gamma^{\prime}$.

Proof. Without excluded coalitions, we have [15]

$$
\varphi_{j}(N, v)=\varphi_{j}^{E}(N, v, \Gamma)>\varphi_{i}^{E}(N, v, \Gamma)=\varphi_{i}(N, v)
$$


$i \in L, j \in R,|R|<|L|$. The players in $R$ obtain the marginal contribution 1 in more rank orders then the players in $L$. Excluding coalitions between $i$ and $j$ reduces the number of admissible rank orders for both players to the same extent; i.e., we have $\left|\Omega^{\prime}(i)\right|-|\Omega(i)|=\left|\Omega^{\prime}(j)\right|-|\Omega(j)|$. The number of rank orders that evoke a marginal contribution 1 is reduced for both players in a proportional way with respect to the initial situation with $\Gamma=\varnothing$. Hence absolutely, player $j$ loses a higher number of rank orders with marginal contribution 1 then player $i$.

Hence, excluding coalitions on asymmetric markets affects the $\varphi^{E}$ payoffs of the scarce side of the market to a higher extend.

\section{Discussion}

In this article, we analyzed properties of the $\varphi^{E}$ value for some classes of games. For further research, the following theoretical lines of development could be interesting. Since a large body of literature deals with axiomatizations of the Shapley value, one possible purpose is to develop new axiomatizations of the $\varphi^{E}$ value and to compare them. In addition, the effects of excluding coalitions could be analyzed in the framework of other structures of cooperative game theory like partitions $[16,17]$, levels $[18,19]$, networks $[3,20]$ or hierarchies [21-23]. Another theoretical development could be a modified version of the $\varphi^{E}$ value that is based on other value-like solution concepts of cooperative game theory [24]. Additionally, the issue of refusing cooperation could be analyzed for value-like solution concepts like the core. One last suggested theoretical development could be the application of the concept of stability [6] to the set of excluded coalitions.

Another line of research is the experimental one. With this research one could check if the payoffs determined by the $\varphi^{E}$ value are realistic and, hence, if the $\varphi^{E}$ value is an appropriate way to model economic situations.

With respect to strategic applications of excluded coalitions [10,11], a two step model could be analyzed. ${ }^{3}$ After the decision on the excluded coalitions (the set of inadmissible coalitions $\Gamma$ ), the players' $\varphi^{E}$ payoff result. For the games analyzed in Section 3, it is a dominant strategy to state $E_{i}=\varnothing$ for all $i \in N$. Hence, we have an equilibrium with $\Gamma=\varnothing$ and the Shapley payoffs result. For further research, an interesting topic could be the analysis of cooperative games with less symmetric assumptions (see [26], for example) and non-cooperative games with negotiations between players after determining their strategies (i.e., sequential decisions on $E_{i}$ ). In particular, our results on the apex game (all minor players are affect adversely if one of them refuse cooperation with $i_{0}$ ) and asymmetric glove markets (the shorter side of the market is more affected than the longer side) indicate fruitful research.

Acknowledgments: I am grateful to two anonymous referees for comments on this paper. I acknowledge support by the German Research Foundation and the Open Access Publication Funds of the TU Dresden.

Conflicts of Interest: The author declares no conflict of interest.

\section{References}

1. Hiller, T. Excluded coalitions and the distribution of power in parliaments. Appl. Econ. 2016, 48, 321-330.

2. Shapley, L.S. A Value for N-Person Games. In Contributions to the Theory of Games; Kuhn, H.W., Tucker, A.W., Eds.; Princeton University Press: Princeton, NJ, USA, 1953; Volume 2, pp. 307-317.

3. Myerson, R.B. Graphs and Cooperation in Games. Math. Oper. Res. 1977, 2, 225-229.

4. Bergantiños, G.; Carreras, F.; Garca-Jurado, I. Cooperation when some players are incompatible. Z. Oper. Res. 1993, 38, 187-201.

5. Alvarez-Mozos, M.; Hellman, Z.; Winter, E. Spectrum value for coalitional games. Games Econ. Behav. 2013, $82,132-142$.

3 For link formation in networks, this strategic model is analyzed by [25]. 
6. Hart, S.; Kurz, M. Endogenous Formation of Coalitions. Econometrica 1983, 51, 1047-1064.

7. Casajus, A. Outside Options, Component Efficiency, and Stability. Games Econ. Behav. 2009, 65, 49-61.

8. Kilgour, D.M. A Shapley Value for Cooperative Games with Quarrelling. In Game Theory as A Theory of $A$ Conflict Resolution; Rapoport, A., Ed.; Theory and Decision Library; Springer: Dordrecht, The Netherlands, 1974; Volume 2, pp. 193-206.

9. Kilgour, D.M. Axioms for Shapley values in games with quarrelling. Theory Decis. 1977, 8, 193-207.

10. Kóczy, L. Strategic Power Indices: Quarrelling in Coalitions; Working Paper; Hungarian academy of Sciences Budapest: Budapest, Hungary, 2008.

11. Kóczy, L. Power Indices When Players can Commit to Reject Coalitions. Homo Oecon. 2016, 33, 77-91.

12. Myerson, R.B. Conference structures and fair allocation rules. Int. J. Game Theory 1980, 9, 169-182.

13. Von Neumann, J.; Morgenstern, O. Theory of Games and Economic Behavior; Princeton University Press: Princeton, NJ, USA, 1944.

14. Montero, M. Non-cooperative bargaining in apex games and the kernel. Games Econ. Behav. 2002, 41, 309-321.

15. Shapley, L.S.; Shubik, M. Pure Competition, Coalitional Power, and Fair Division. Int. Econ. Rev. 1969, 10, 337-362.

16. Aumann, R.J.; Drèze, J.H. Cooperative Games with Coalition Structures. Int. J. Game Theory 1974, 3, $217-237$.

17. Owen, G. Values of Games with a Priori Unions. In Essays in Mathematical Economics and Game Theory; Henn, R., Moeschlin, O., Eds.; Springer: Berlin, Germany, 1977; pp. 76-88.

18. Kalai, E.; Samet, D. On Weighted Shapley Values. Int. J. Game Theory 1987, 16, 205-222.

19. Winter, E. A Value for Cooperative Games with Levels Structure of Cooperation. Int. J. Game Theory 1989, 18, 227-240.

20. Borm, P.; Owen, G.; Tijs, S. On the Position Value for Communication Situations. SIAM J. Discret. Math. 1992, 5, 305-320.

21. Gilles, R.P.; Owen, G.; van den Brink, R. Games with Permission Structures: The Conjunctive Approach. Int. J. Game Theory 1992, 20, 277-293.

22. Van den Brink, R.; Gilles, R.P. Axiomatizations of the Conjunctive Permission Value for Games with Permission Structure. Games Econ. Behav. 1996, 12, 113-126.

23. Van den Brink, R. An Axiomatization of the Disjunctive Permission Value for Games with a Permission Structure. Int. J. Game Theory 1997, 26, 27-43.

24. Banzhaf, J.F. Weighted Voting Doesn't Work: A Mathematical Analysis. Rutgers Law Rev. 1965, 19, $317-343$.

25. Dutta, B.; van den Nouweland, A.; Tijs, S. Link formation in cooperative situations. Int. J. Game Theory 1998, $27,245-256$.

26. Laruelle, A.; Valenciano, F. A Critical Reappraisal of Some Voting Power Paradoxes. Public Choice 2005, $125,17-41$.

(C) 2018 by the authors. Licensee MDPI, Basel, Switzerland. This article is an open access article distributed under the terms and conditions of the Creative Commons Attribution (CC BY) license (http://creativecommons.org/licenses/by/4.0/). 